\title{
The visual line spectrum of AG Pegasi in 1995
}

\author{
N.A. Tomov ${ }^{1}$, M.T. Tomova ${ }^{1}$, and D.V. Raikova ${ }^{2}$ \\ 1 National Astronomical Observatory Rozhen, P.O. Box 136, BG-4700 Smolyan, Bulgaria \\ e-mail: rozhen@tu-plovdiv.bg \\ ${ }^{2}$ Institute of Astronomy, Blvd. Tzarigradsko shosse 72, BG-1784 Sofia, Bulgaria
}

Received April 24; accepted October 16, 1997

\begin{abstract}
Intermediate resolution observations of the blue spectrum of the symbiotic star AG Peg, being at a colliding wind stage in its evolution with decreasing luminosity and mass-loss rate of its hot component, have been realized. Profiles, fluxes and radial velocity data have been obtained. They are compared with earlier data, taken approximately at the same orbital phases. It turns out that the radial velocities of many lines differ from these data which is probably determined by a change of dynamics of the nebula. The comparison shows, also, the intensity of all emission lines has decreased, but the velocity of the wind of the hot component is invariable. The fluxes of the lines appearing in this wind have been theoretically calculated by means of values of the mass-loss rate obtained on the basis of contemporaneous UV observations. They are in good agreement with the observed fluxes which is a confirmation of this mass-loss rate. The intensity decrease of the narrow emission lines is discussed.
\end{abstract}

Key words: binaries: symbiotic — stars: individual: AG Peg — stars: mass-loss — line: profiles

\section{Introduction}

The symbiotic binary systems possess dense gaseous nebulae formed, in most cases, by the wind of their cool components. At some stages of their evolution, however, these nebulae are formed by the winds of the two components. The system AG Peg (HD 207757) is the oldest known symbiotic nova. It has undergone a single outburst (Lundmark 1921; Boyarchuk 1967), which is the most prolonged one among the outbursts of symbiotics. Its visual light was $9^{\mathrm{m}}$ before the middle of the last century. Between the years 1841 and 1855 it has begun to increase, reaching a maximum of approximately $6^{\mathrm{m}}$ around 1885 . Then it has gradually decreased and now it is practically equal to its value before the outburst.

Send offprint requests to: N.A. Tomov
The analysis of the observations carried out in different spectral regions during the past years, imply one and the same model of this system - a stage of colliding winds. For the first time this model was proposed by Penston \& Allen (1985) on the basis of three IUE high resolution spectra, where a high velocity $\mathrm{P}$ Cyg wind was detected in the lines of N V, N IV, C IV and He II. Since the cool giant also loses mass through a stellar wind, it was concluded that the two winds probably interact.

Later the UV spectrum of AG Peg was studied by Kenyon et al. (1993), Vogel \& Nussbaumer (1994) and Altamore \& Cassatella (1997). Each of these analyses showed that a hot high velocity wind is observed in the lines of Nv, C IV and He II and in every one of them a conclusion about colliding winds was made.

A model of winds in collision was proposed also by Tomov (1993b) on the basis of profiles, fluxes and radial velocities, derived from homogeneous high dispersion spectral observations in the visual during two consecutive orbital cycles (Tomov \& Tomova 1992; Tomov 1993a). The broad components of the first Balmer lines and the line He II 4686 were caused by radial flow of gas with high velocity.

Supposing a regime of colliding winds in the AG Peg system Vogel \& Nussbaumer (1994) theoretically calculated the radio image of its nebula being in good agreement with the observed one. A proof for existence of a shock region of the winds was obtained on the basis of ROSAT data by Mürset et al. (1995). They created a hydrodynamical model whose X-ray energy distribution was in agreement with the observations.

There are also observational data showing that some parameters of the wind of the hot companion are changed. According to both UV (Kenyon et al. 1993; Vogel \& Nussbaumer 1994; Altamore \& Cassatella 1997) and visual (Hutchings \& Redman 1972; Ilmas 1987; Tomov \& Tomova 1992) observations, the lines, appearing in this wind, began to weaken after the year 1978. The decrease of their intensities is determined by decreasing massloss rate (Kenyon et al. 1993; Vogel \& Nussbaumer 1994; 

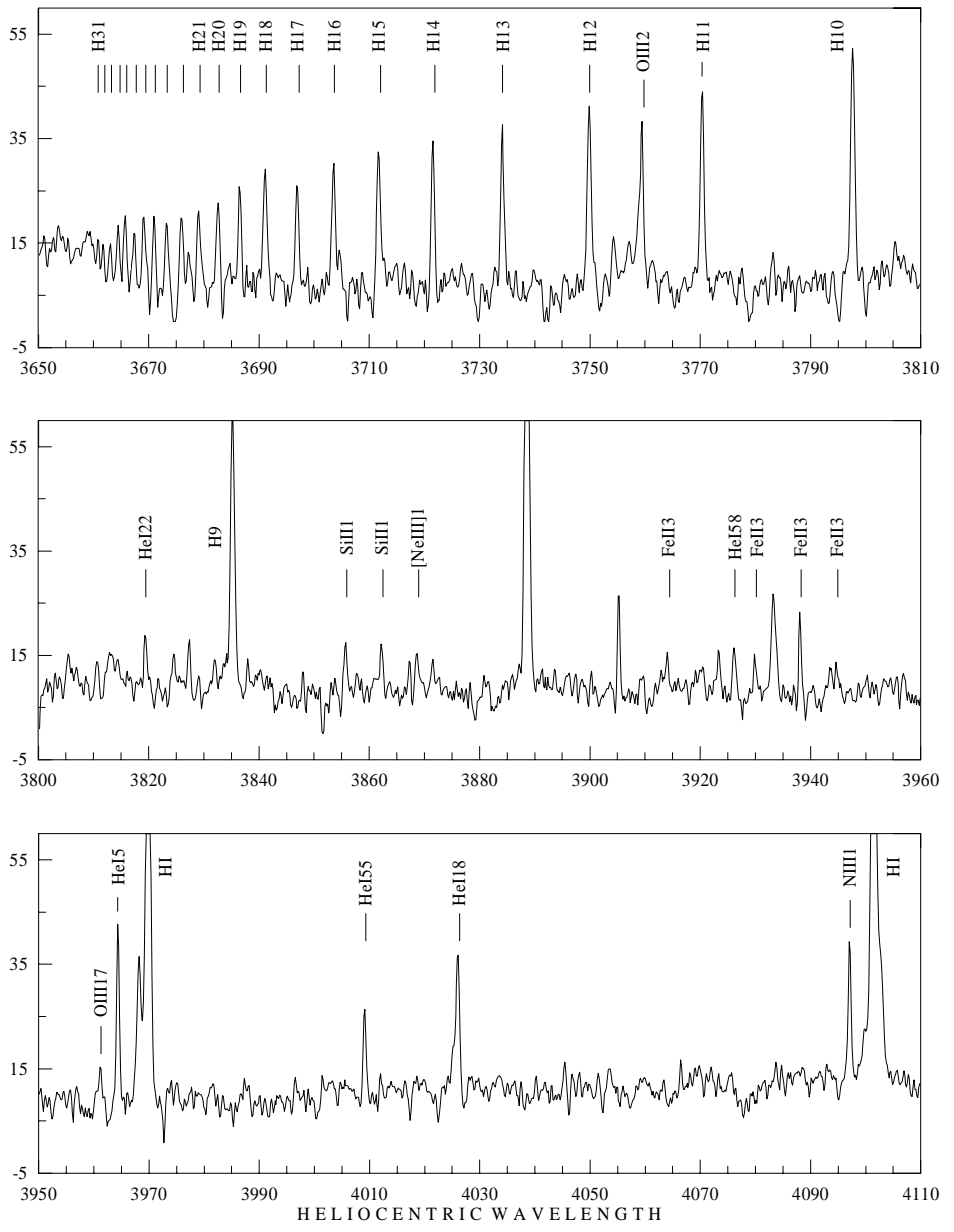

Fig. 1. Sections of the spectrum, taken on JD 2449969.38 . The ordinate scale is in arbitrary units

Altamore \& Cassatella 1997). Having in mind their behaviour Zamanov \& Tomov (1995) prognosticated that in the near future the change of the mass-loss at the observed rates will cause its decreasing below a given minimal value. At that time the momentum of the hot wind will not be sufficient for realizing a collision and the system will move to a regime of a wind accretion. This transformation will be the final stage of the outburst begun in the first half of the last century.

The change of the mass-loss rate of the hot companion causes changes in the dynamics and the physical parameters of the nebular envelope and consequently of its spectrum. That is why we observed the blue region where most of the visual emission lines are situated. In this paper we analyse and discuss intermediate resolution observations of AG Peg taken in 1995 and compare them with the observations in the middle of the last decade (Tomov \& Tomova 1992; Tomov 1993a) to study the evolution of the visual emission lines during the final stage of the outburst.

\section{Observations and reduction}

The present work is based on two spectrograms, taken with the Coudé spectrograph of the $2 \mathrm{~m}$ RCC telescope of the National Astronomical Observatory Rozhen (NAO) on Sep. 8, 1995 (JD 2449 969.38) and Sep. 13, 1995 (JD 2449 974.38). The observations were performed in the region from $3600 \AA$ to $5000 \AA$ on ORWO ZU emulsion, with a resolution of $0.5 \AA$. (The reciprocal linear dispersion was $18 \AA \mathrm{mm}^{-1}$.) Both exposures were comparatively deep, each of 240 minutes, in order to bring out the wings of the bright emission lines and to detect the fainter features. The spectra were digitized with the Joyce Loebl microdensitometer of NAO and ReWiA package was used for wavelength and density calibration as well as for calculation of the radial velocities and the equivalent widths. Sections of the spectrum taken on JD 2449969.38 are displayed in Fig. 1 where the lines with measured radial velocities have been marked. As the strongest lines are not seen in this figure, the profile of one of them is displayed separately in Fig. 2. The profiles of the others are similar. The radial velocity of each group of lines is listed in Table 1. 

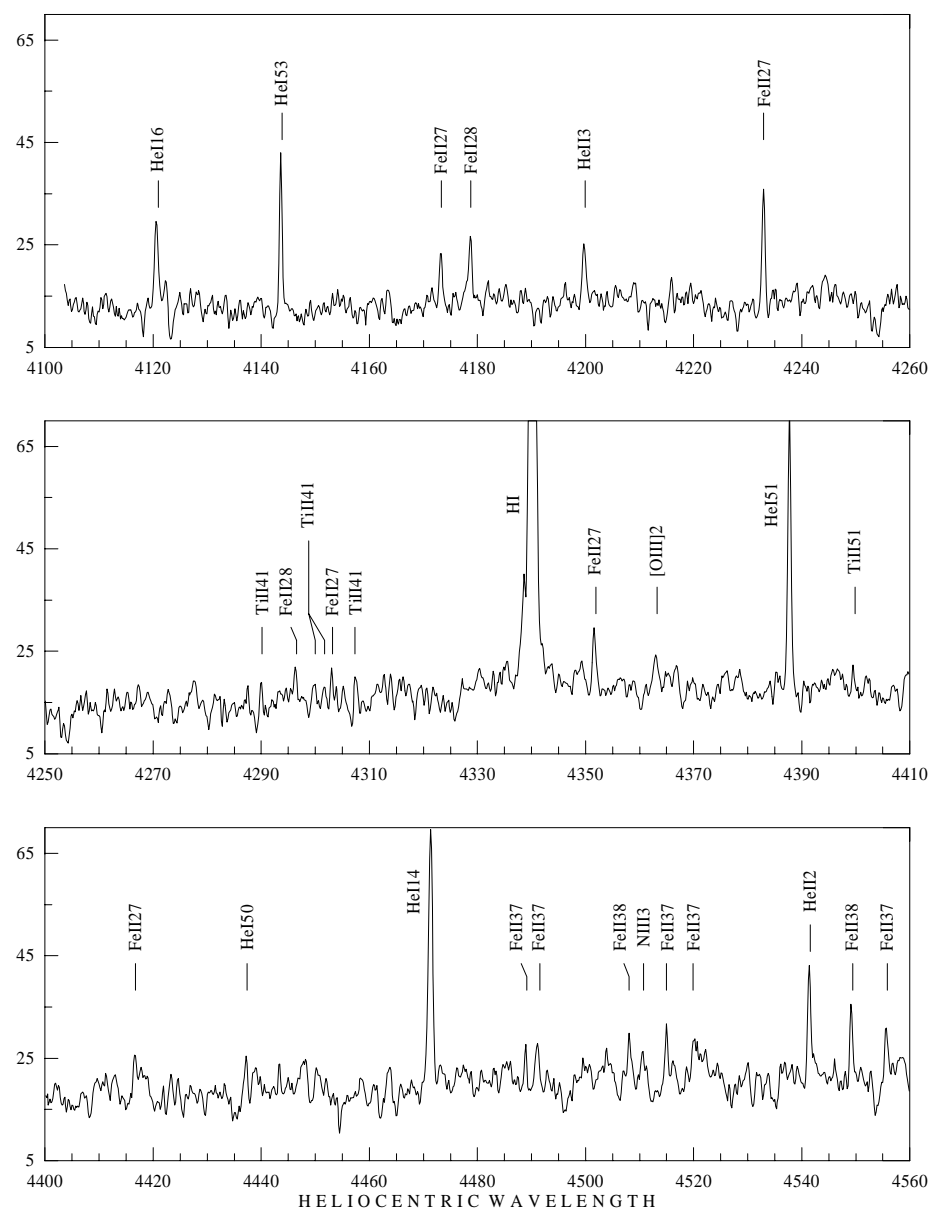

Fig. 1. continued

Table 1. Radial velocities in units of $\mathrm{km} \mathrm{s}^{-1}$

\begin{tabular}{|c|c|c|c|c|c|}
\hline \multirow[t]{2}{*}{ Elem. } & \multicolumn{2}{|c|}{ JD $2449000^{\mathrm{d}}+$} & \multirow[t]{2}{*}{ Elem. } & \multicolumn{2}{|c|}{ JD $2449000^{\mathrm{d}}+$} \\
\hline & 969.38 & 974.38 & & $969^{\mathrm{d}} \cdot 38$ & $974^{\mathrm{d}} .38$ \\
\hline Abs. lines & $-18.1 \pm 2.9$ & $-19.5 \pm 1.8$ & He II, N III, C III & $-20.8 \pm 2.4$ & $-20.3 \pm 0.9$ \\
\hline H I & $-22.2 \pm 1.0$ & $-20.1 \pm 1.4$ & HeII $4686(\mathrm{~N})$ & -13.7 & -6.5 \\
\hline $\mathrm{He}^{1} \mathrm{I}$ & $-14.2 \pm 1.0$ & $-14.4 \pm 1.8$ & [O III] 4363 & -16.1 & -18.7 \\
\hline $\mathrm{He}^{3} \mathrm{I}$ & $-14.1 \pm 1.6$ & $-17.0 \pm 0.3$ & Fe II, Ti II & $-18.7 \pm 0.6$ & $-18.2 \pm 0.5$ \\
\hline
\end{tabular}

We obtained the line fluxes using only the spectrogram of JD 2449969.38 as it turned out that the sensitivity of the other one was decreased in the long wavelengths region. Only fluxes of comparatively stronger lines were measured such that their equivalent width errors should be less than $30 \%$. We consider the intensities of the same emission lines investigated in the work of Tomov (1993a) to have possibility for comparison. Some of these lines are the members of Pickering series of He II having wavelengths $\lambda \lambda 4200$ and $4542 \AA$. They are unresolved blends. At the phases of our observations the line He II 4542 is blending with the line Fe II 4542 , but the latter of them has considerably lower intensity. The line He II 4200 is blending with the line N III 4200 which has also lower intensity. In our opinion the blending of these lines works unessentially on the measurement of their equivalent widths.

The line fluxes have been obtained by means of our unpublished $B=9{ }^{\mathrm{m}} 76$ and $V=8^{\mathrm{m}} 56$ photometric estimates, taken on JD 2449974.34 . The monochromatic continuum fluxes at the positions of emission lines considered were calculated via linear interpolation of the fluxes at the positions of the sensitivity maxima of the $B$ and $V$ 

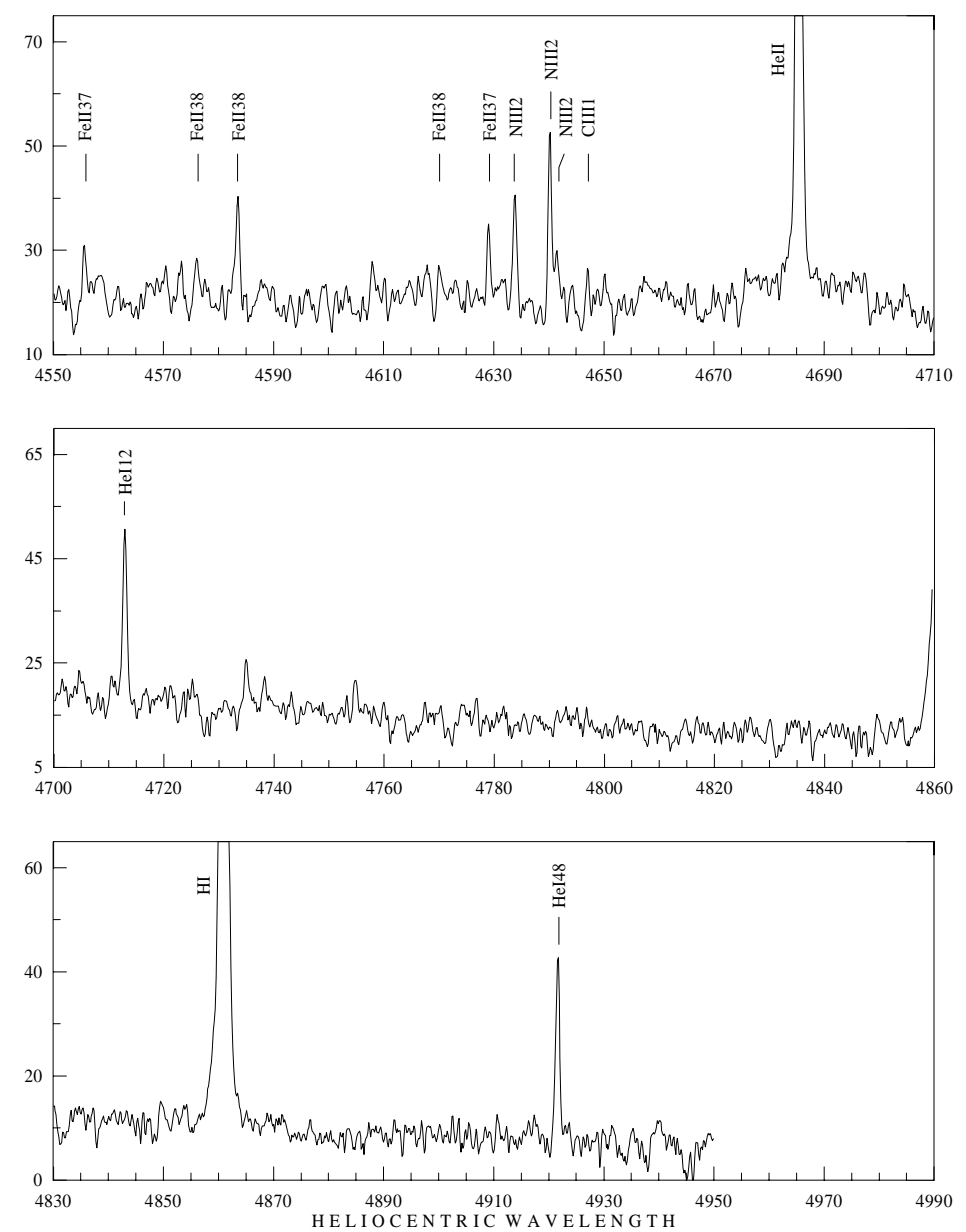

Fig. 1. continued

Table 2. Emission line fluxes in units of $10^{-11} \mathrm{erg} \mathrm{cm}^{-2} \mathrm{~s}^{-1}$

\begin{tabular}{|c|c|c|c|c|c|c|c|c|}
\hline Line & 1986 & 1995 & Line & 1986 & 1995 & Line & 1986 & 1995 \\
\hline N III 4097 & 0.43 & 0.19 & Fe II 4296 & 0.06 & 0.03 & Fe II 4584 & 0.14 & 0.14 \\
\hline $\mathrm{H} \delta 4103$ & 4.95 & 1.52 & $\mathrm{H} \gamma 4340(\mathrm{~N})$ & 8.07 & 2.00 & Fe II 4629 & 0.15 & 0.10 \\
\hline $\mathrm{He}^{3} \mathrm{I} 4121$ & 0.32 & 0.16 & [O III] 4363 & 0.34 & 0.07 & N III 4634 & 0.19 & 0.15 \\
\hline $\mathrm{He}^{1} \mathrm{I} 4144$ & 0.49 & 0.17 & $\mathrm{He}^{1} \mathrm{I} 4388$ & 0.59 & 0.36 & N III 4640 & 0.35 & 0.21 \\
\hline Fe II 4173 & 0.11 & 0.07 & $\mathrm{He}^{3} \mathrm{I} 4471$ & 0.71 & 0.38 & HeII $4686(\mathrm{~N})$ & 5.20 & 1.74 \\
\hline Fe II 4179 & 0.12 & 0.09 & Fe II 4508 & 0.09 & 0.04 & $\mathrm{He}^{3} \mathrm{I} 4713$ & 0.53 & 0.29 \\
\hline He II 4200 & 0.10 & 0.08 & Fe II 4520 & 0.09 & & $\mathrm{H} \beta 4861(\mathrm{~N})$ & 14.71 & 6.02 \\
\hline Fe II 4233 & 0.23 & 0.11 & He II 4542 & 0.12 & 0.12 & $\mathrm{He}^{1} \mathrm{I} 4922$ & 1.18 & 0.65 \\
\hline
\end{tabular}

photometric systems. The error of the monochromatic continuum fluxes, obtained in this way, is due first of all to the presence of the bands of titanium oxide. This error is approximately equal to the error of the level of the local continuum ( $\sim 10 \%)$, as titanium oxide bands of the AG Peg system are shallow because of an overwhelming by the hot continuum (Boyarchuk 1966; Tomov \& Tomova 1992). The line fluxes were corrected for interstellar reddening. There are several values for the excess $E(B-V)$, obtained by different methods and placed in the range $0.08-0.15$ (Penston \& Allen 1985). We adopted the value $E(B-V)=0.12$ and used the extinction law by Seaton (1979). The line fluxes are listed in Table 2.

For the aims of our consideration the ephemeris of Fernie (1985) $\operatorname{Max}(V)=$ JD $2442710.1+816.5 \times E$ will be used. The epoch of the photometric maximum when the hot companion is before the giant is used as a start 
Table 3. Radial velocity data of AG Peg in units of $\mathrm{km} \mathrm{s}^{-1}$ (Tomov \& Tomova 1992)

\begin{tabular}{|c|c|c|c|c|c|c|c|c|}
\hline $\begin{array}{r}\mathrm{JD}_{\odot} \\
2446000+\end{array}$ & Phase & Abs.lines & $\begin{array}{r}\text { He II } 4686 \\
\text { (B) }\end{array}$ & $\mathrm{HI}$ & $\mathrm{He}^{1} \mathrm{I}$ & $\begin{array}{r}\text { He II, N III, } \\
\text { O III, C III }\end{array}$ & $\begin{array}{r}\text { HeII } 4686 \\
(\mathrm{~N})\end{array}$ & [O III] 4363 \\
\hline 221.50 & 0.300 & $-19.8 \pm 0.7$ & -0.6 & $-9.2 \pm 2.0$ & $-12.4 \pm 0.9$ & $-12.6 \pm 2.3$ & -6.8 & -21.1 \\
\hline 244.55 & 0.329 & $-21.0 \pm 2.0$ & & $-20.2 \pm 1.0$ & $-17.4 \pm 1.1$ & $-23.5 \pm 2.0$ & -22.3 & -31.4 \\
\hline 252.52 & 0.338 & $-18.3 \pm 1.4$ & & $-17.0 \pm 0.2$ & $-14.4 \pm 0.3$ & $-14.6 \pm 2.2$ & -11.8 & -29.5 \\
\hline 252.57 & 0.339 & $-22.0 \pm 3.4$ & -6.7 & $-14.2 \pm 0.4$ & $-13.7 \pm 1.5$ & $-20.2 \pm 1.3$ & -21.7 & -29.9 \\
\hline 273.52 & 0.364 & $-18.7 \pm 1.0$ & 3.0 & $-31.2 \pm 1.6$ & $-19.3 \pm 0.4$ & $-21.7 \pm 1.6$ & -25.3 & -36.6 \\
\hline 277.40 & 0.369 & $-22.0 \pm 1.8$ & & $-35.6 \pm 1.6$ & $-19.1 \pm 0.9$ & $-23.4 \pm 1.8$ & -19.5 & -33.7 \\
\hline 301.39 & 0.398 & $-21.3 \pm 1.7$ & & $-38.4 \pm 2.4$ & $-20.0 \pm 1.5$ & $-29.0 \pm 4.1$ & -29.3 & -35.0 \\
\hline 330.23 & 0.434 & $-16.7 \pm 0.7$ & -35.8 & $-41.0 \pm 6.8$ & $-29.1 \pm 2.7$ & $-32.7 \pm 0.1$ & -34.6 & -40.2 \\
\hline 370.30 & 0.483 & $-19.1 \pm 1.3$ & -32.7 & $-53.6 \pm 5.6$ & $-30.4 \pm 0.6$ & $-35.4 \pm 0.9$ & -43.1 & -42.3 \\
\hline 452.21 & 0.583 & & & $-64.9 \pm 1.4$ & $-31.1 \pm 0.4$ & $-41.9 \pm 2.9$ & -53.9 & -45.5 \\
\hline 603.53 & 0.768 & $-12.8 \pm 1.7$ & -36.8 & $-20.5 \pm 1.2$ & $-19.4 \pm 1.9$ & $-20.0 \pm 1.7$ & -26.3 & -25.7 \\
\hline 603.57 & 0.768 & $-14.9 \pm 0.9$ & -47.4 & $-16.5 \pm 1.2$ & -21.3 & $-16.6 \pm 1.6$ & -22.6 & -30.1 \\
\hline 605.49 & 0.771 & $-16.5 \pm 0.7$ & & $-24.2 \pm 1.1$ & $-19.1 \pm 0.5$ & $-19.9 \pm 1.4$ & -27.8 & -20.2 \\
\hline 627.53 & 0.798 & $-12.7 \pm 1.4$ & -41.7 & $-16.4 \pm 0.7$ & $-15.4 \pm 0.5$ & $-17.4 \pm 0.3$ & -24.1 & -22.7 \\
\hline 630.19 & 0.801 & $-12.8 \pm 0.9$ & -50.4 & $-17.3 \pm 1.2$ & $-14.1 \pm 0.4$ & $-16.3 \pm 0.5$ & -20.2 & -16.2 \\
\hline 638.49 & 0.811 & $-13.8 \pm 1.2$ & -43.3 & $-14.2 \pm 0.3$ & $-16.2 \pm 0.8$ & $-19.3 \pm 2.3$ & -25.0 & -19.8 \\
\hline 638.59 & 0.811 & $-16.3 \pm 1.1$ & & $-16.9 \pm 0.6$ & $-17.6 \pm 1.3$ & $-15.6 \pm 0.5$ & -16.7 & -17.7 \\
\hline 660.53 & 0.838 & $-14.8 \pm 1.0$ & & $-15.8 \pm 0.6$ & $-14.4 \pm 1.2$ & $-15.9 \pm 1.8$ & -21.0 & -14.1 \\
\hline 752.18 & 0.950 & $-21.1 \pm 0.9$ & & $-2.9 \pm 0.4$ & $-11.1 \pm 0.4$ & $-7.8 \pm 1.0$ & 0.9 & 7.6 \\
\hline 755.27 & 0.954 & $-19.2 \pm 1.0$ & -30.9 & $-7.8 \pm 0.3$ & $-14.7 \pm 0.5$ & $-8.4 \pm 1.4$ & -2.8 & 2.1 \\
\hline 775.15 & 0.979 & $-19.2 \pm 1.9$ & -28.6 & $-11.0 \pm 0.8$ & $-8.6 \pm 0.4$ & $-6.6 \pm 1.0$ & 5.8 & 14.8 \\
\hline 928.50 & 0.166 & $-26.2 \pm 1.4$ & 32.3 & $-7.8 \pm 3.7$ & $-15.5 \pm 0.4$ & $-8.4 \pm 0.9$ & -3.1 & -4.0 \\
\hline 990.52 & 0.242 & $-22.0 \pm 1.2$ & 10.5 & $-14.0 \pm 0.7$ & $-20.2 \pm 1.2$ & $-15.3 \pm 2.4$ & 2.8 & -37.6 \\
\hline 991.51 & 0.244 & $-24.2 \pm 1.3$ & & $-13.1 \pm 1.8$ & $-18.1 \pm 1.0$ & $-15.7 \pm 0.4$ & -9.4 & -31.7 \\
\hline 1023.53 & 0.283 & $-22.7 \pm 2.0$ & & $-18.8 \pm 0.9$ & $-16.4 \pm 0.8$ & $-13.6 \pm 0.4$ & -14.0 & -33.3 \\
\hline 1025.48 & 0.285 & $-21.6 \pm 1.1$ & & $-17.0 \pm 1.0$ & $-15.8 \pm 1.1$ & $-14.8 \pm 0.6$ & -22.3 & -33.2 \\
\hline 1047.26 & 0.312 & $-22.0 \pm 1.9$ & 13.8 & $-15.5 \pm 0.3$ & $-14.2 \pm 0.7$ & $-15.5 \pm 0.4$ & -16.7 & -34.9 \\
\hline 1346.50 & 0.678 & & & $-34.6 \pm 3.6$ & $-18.1 \pm 0.2$ & $-26.3 \pm 2.0$ & -40.7 & -31.8 \\
\hline
\end{tabular}

epoch. In this case our observations are at phases 0.891 and 0.897 .

The data obtained will be compared with the results of our previous observations (Tomov \& Tomova 1992) and that is why some of these results are listed in Table 3 and displayed in Fig. 3 as well. These are radial velocities data derived also on the basis of photographic observations in the blue spectral region. $40 \%$ of these spectrograms were taken with the Coudé spectrograph of the $6 \mathrm{~m}$ telescope of the Special Astrophysical Observatory of Russian Academy of Sciences and have dispersion of $9 \AA \mathrm{mm}^{-1}$ and the rest - with the Coudé spectrograph of the $2 \mathrm{~m}$ RCC telescope of NAO, with dispersion of $18 \AA \mathrm{mm}^{-1}$. All these spectrograms were processed with the oscilloscopic comparator of NAO, whose positioning error is equal to $0.5 \mu \mathrm{m}$.

The radial velocity curves of all groups of emission lines (Fig. 3) were plotted by means of the same program, used for obtaining the orbital solution. The lines in this figure plot the best-fitting circular orbits for the data in Table 3. The phase shift of each curve with respect to the absorption lines velocity curve, determined by the orbital
Table 4. Parameters of the radial velocities curves of AG Peg

\begin{tabular}{lcccc}
\hline Group of lines & $\begin{array}{c}\text { Phase } \\
\text { shift }\end{array}$ & $\begin{array}{c}V_{0} \\
\left(\mathrm{~km} \mathrm{~s}^{-1}\right)\end{array}$ & $\begin{array}{c}K \\
\left(\mathrm{~km} \mathrm{~s}^{-1}\right)\end{array}$ & $\begin{array}{c}s \\
\left(\mathrm{~km} \mathrm{~s}^{-1}\right)\end{array}$ \\
\hline Abs. lines & & -18.37 & 5.22 & 1.60 \\
He II 4686 (B) & 0.50 & -20.69 & 31.77 & 9.88 \\
H I & 0.38 & -18.56 & 27.94 & 6.88 \\
He $^{1}$ I & 0.38 & -16.96 & 9.01 & 3.91 \\
He II, N III, O III, C III & 0.38 & -16.74 & 19.18 & 3.14 \\
He II 4686 (N) & 0.40 & -18.00 & 27.36 & 5.12 \\
[O III] 4363 & 0.34 & -22.81 & 24.08 & 6.83 \\
\hline
\end{tabular}

motion of the cool component, is presented in the second column of Table 4 . The parameters corresponding to the baricentric velocity $V_{0}$ and the semiamplitude $K$ for each curve are presented in the third and fourth columns of this table. The standard deviation $s$ is in the last column. 


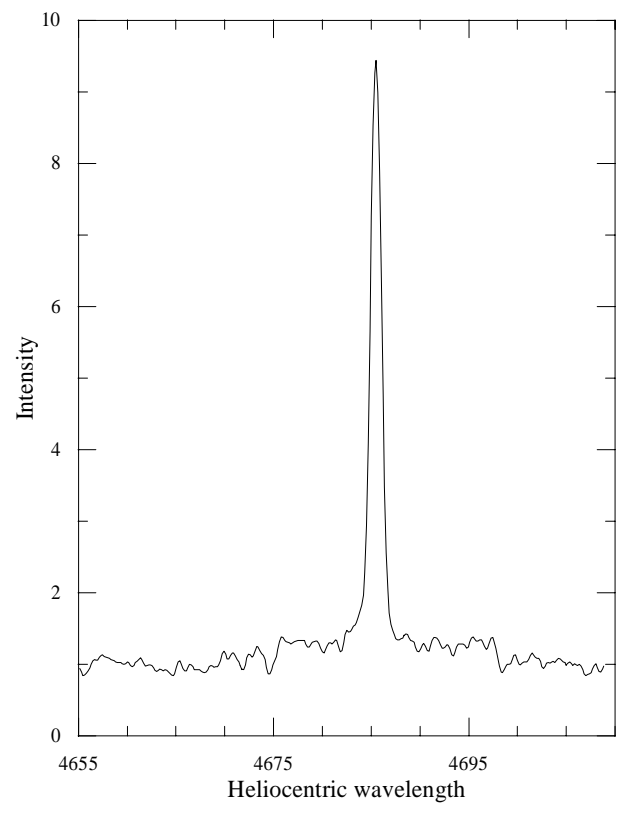

Fig. 2. The profile of the HeII 4686 line, observed on JD 2449969.38 and normalized with respect to the local continuum. It consists of two components - a narrow and a broad one

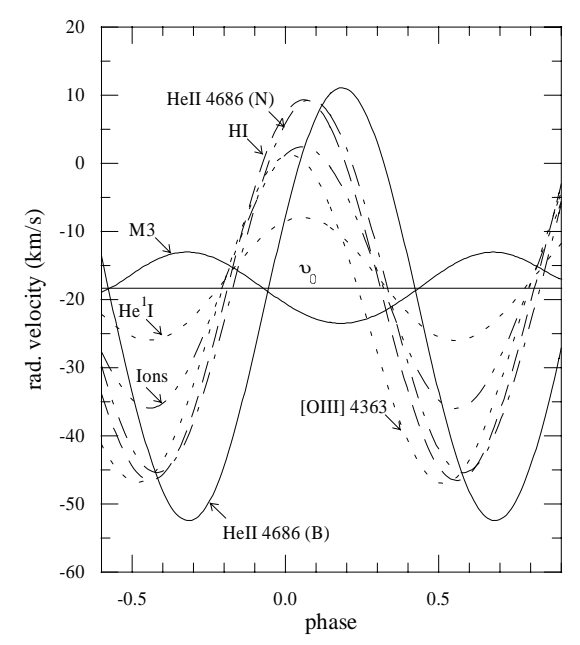

Fig. 3. The orbital radial velocity curves of the visual lines of AG Peg from the work of Tomov \& Tomova (1992). The elements of each curve as well as the designation of the M 3 giant and the barycentric velocity have been marked

\section{Description and analysis of the emission line spectrum}

\subsection{The Balmer lines}

The lines of Balmer series are visible as far as H31. The profiles of the first of them consist of two components: a central narrow component with FWHM equal to about $95 \mathrm{~km} \mathrm{~s}^{-1}$ and a broad component, indicating stellar wind and having FWZI (full width of zero intensity) of about
$2000 \mathrm{~km} \mathrm{~s}^{-1}$. Only the broad component of $\mathrm{H} \beta$ is confidently seen. The profiles of the rest of the Balmer members consist of one component, i.e. they are ordinary nebular lines. Some of the Balmer profiles have asimmetry.

The line H 8 is badly blending with the line He I 3889 and is inappropriate for investigation.

We obtained radial velocities, measuring only the narrow components (Table 1 ). The radial velocities of all lines were the same within the range of the error, i.e. we didn't ascertain the existence of Balmer progression. The velocities of both spectra differ appreciably from the values of the radial velocity curve of the hydrogen lines at our phases in Fig. 3. These differences exceed the level of our error and that is why we consider they are due to a change of dynamics of the nebula.

The line fluxes (Table 2) obtained at the present time are compared with the fluxes obtained in 1986 (Tomov 1993a). Each of the latter of them is an arithmetical mean of three spectra taken at the moments JD 2446660.53 , 2446752.18 and 2446755.27 , which are at phases 0.838 , 0.950 and 0.954 . We compare spectra taken approximately at the same phases since the intensity of most of the emission lines vary during the orbital cycle. The line fluxes in the work of Tomov (1993a) have been systematically reduced by a factor of about 1.5. The data used in the present work have been improved and then deredened in the way described in the last section. The data from the table show that the fluxes of the Balmer lines have decreased on average by a factor of 3.2 .

\subsection{The helium lines}

The FWHM of the singlets is $45-50 \mathrm{~km} \mathrm{~s}^{-1}$ and those of the triplets - about $60 \mathrm{~km} \mathrm{~s}^{-1}$. Some of the lines of both groups have asymmetry in their profiles. The two groups have the same velocity (Table 1) practically equal to the value at our phases of the radial velocity curve of the helium lines in Fig. 3. The line fluxes of the singlets have decreased on average by a factor of 2.1 and those ones of the triplets - by a factor of 1.9 (Table 2).

\subsection{The lines of elements of high degree of ionization}

Lines of different highly ionized elements namely HeII, $\mathrm{N}$ III, O III and C III are present in the blue region of the spectrum of AG Peg.

Like the Balmer lines the line of He II 4686 consist of two components: a central nebular component with FWHM equal to $80 \mathrm{~km} \mathrm{~s}^{-1}$ and a broad component, indicating stellar wind. The profile of the broad component, observed at the moment JD 2446755.27 when the line was more intensive is compared with the one, observed at JD 2449969.38 in Fig. 4. The error of the local continuum in the first case is equal to $\pm 5 \%$ as Kodak IIaO emulsion having a lower noise was used. This error in the second case is about $\pm 10 \%$. The observed spectrum in 
the region of the He II 4686 broad component was corrected through removing the strongest absorption lines of the red giant spectrum. Then the individual profiles were analysed by fitting with a sum of two gaussian components. The FWHM of the broad component in the first case is equal to $1210 \pm 50 \mathrm{~km} \mathrm{~s}^{-1}$ and in the second one $-1310 \pm 200 \mathrm{kms}^{-1}$. Taking into account the observational errors we have no reason to suppose that the line width has changed. This procedure allows us to obtain the equivalent width and the line flux with errors equal to about $30 \%$ in the first case, and about $50 \%$ in the second one. These errors are due first of all to the errors of the local continuum. If we take a velocity of the wind at a distance $2 \sigma$ from the center of the line this velocity is obtained to be $1030 \mathrm{~km} \mathrm{~s}^{-1}$ and $1110 \mathrm{~km} \mathrm{~s}^{-1}$ in the two cases. We are inclined to adopt the first one of these values made up to $1000 \mathrm{~km} \mathrm{~s}^{-1}$, as it is based on emulsion with better quality.

The velocity of the narrow component of He II 4686 (Table 1) is in agreement with the radial velocity curve of this line in Fig. 3. In a period of nine years its flux has decreased by a factor of 3.0 (Table 2).

We considered only the lines He II 4200 and He II 4542 of the Pickering series as the rest of them are badly blended with Balmer lines. The profiles of the lines He II 4200 and He II 4542 are asymmetric and their FWHM are equal to $55 \mathrm{~km} \mathrm{~s}^{-1}$ and $48 \mathrm{kms}^{-1}$ respectively. The FWHM of the N III lines are about $45-50 \mathrm{~km} \mathrm{~s}^{-1}$.

Since there was a great difference between the velocities of the O III lines of the two spectrograms, their values were excluded from further consideration. The lines of the rest of the elements have practically the same velocities whose arithmetical mean is shown in Table 1 . However, they differ from the values of the radial velocity curve of the ionized elements at the some phases in Fig. 3.

The fluxes of the Pickering lines of He II have decreased on average by a factor of 1.1 and those of N III - by a factor of 1.7 .

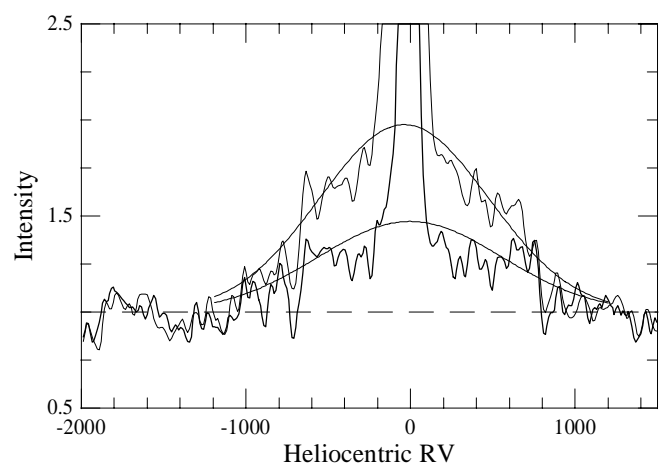

Fig. 4. The broad component of the HeII 4686 line, observed at the moments JD 2446755.27 (more intensive one) and JD 2449969.38 . The level of the local continuum is marked with a dashed line. The radial velocities are in units $\mathrm{km} \mathrm{s}^{-1}$

\subsection{The forbidden lines}

Only the lines of the elements [O III] and [Ne III] are included in this group. In contrast to the middle of eighties (Tomov \& Tomova 1992), now the lines of [O III] $\lambda \lambda 4959$ and 5007 have disappeared entirely and [O III] 4363 has considerably weakened - its flux has been reduced by a factor of 4.9 (Table 2). Its FWHM is equal to $73 \mathrm{~km} \mathrm{~s}^{-1}$.

There was a great difference between the [Ne III] 3868 line radial velocities in the two spectrograms and that is why they were excluded from further consideration. Like H I, HeII, N III and C IV lines, the velocity of the [O III] 4363 line (Table 1) differs considerably from the one obtained previously at the same phases (Fig. 3).

\subsection{The lines of ionized metals}

Besides all groups of lines examined up to now, emission lines of singly ionized and neutral metals as Fe II, Ti II, Si II, CaII, Cr II, Mg II, VII, Ni II, Aliı, Fe I, Si I, Ca I and [Fe II] present in the visual spectrum of AG Peg. Only the Fe II and Ti II lines are intensive enough to make investigation possible. Their FWHM is equal to $45 \mathrm{~km} \mathrm{~s}^{-1}$. The $\mathrm{K}$ and $\mathrm{H}$ lines of $\mathrm{Ca}$ II are inappropriate for investigation because of blending with the lines N III 3934 and He II 3968, [Ne III] 3968 respectively.

The radial velocity of the Fe II and Ti II lines (Table 1) was obtained to be equal to the velocity of the lines of the singly ionized metals (Tomov \& Tomova 1992) which doesn't vary with the orbital phase and is equal to $-18.1 \mathrm{~km} \mathrm{~s}^{-1}$. These facts are probably due to the following reason: in contrast to the rest of nebular lines the lines of the singly ionized metals are excited in a part of the giant chromosphere, which faces the hot companion (Boyarchuk 1966; Kenyon et al. 1993) and is located very close to the mass center of the system, whose velocity is derived in the interval $16-18 \mathrm{~km} \mathrm{~s}^{-1}$ (Hutchings et al. 1975; Tomov \& Tomova 1992; Kenyon et al. 1993).

The line fluxes of Fe II have decreased on average by a factor of 1.7 (Table 2). We assume the part of the giant atmosphere which is ionized by the hot companion has decreased approximately in the same ratio.

\section{The broad components and the mass-loss rate}

The evolution of the hot component of the AG Peg system has been considered by Mürset et al. (1991), Kenyon et al. (1993), Vogel \& Nussbaumer (1994) and Altamore \& Cassatella (1997). Their analyses show that after the year 1978 the radius, the luminosity and the mass-loss rate of this star have been decreased at nearly constant temperature. We will examine if the variations of the line intensities, observed by us, are in line with the variations of these parameters. Only the broad components of the lines $\mathrm{H} \beta, \mathrm{H} \gamma$ and $\mathrm{He}$ II 4686 will be considered since only they appear in the wind of the hot companion (see Sect. 5) and depend solely on its parameters. The fluxes of these 
lines will be calculated and compared with the observed ones.

The mass-loss rate has been derived by Vogel \& Nussbaumer (1994) and Altamore \& Cassatella (1997) with the same methods using UV spectra obtained during approximately one and the same period of time. The first of these methods is based on the dependence of the equivalent width of the He II 1640 line on the mass-loss rate of the Wolf-Rayet stars (Schmutz et al. 1989) and is related to the case when the line is optically thick. This dependence has been extrapolated to the atmosphere of the hot companion of AG Peg. The second method is based on the relation between the energy emitted in the He II 1640 line and the mass-loss rate when the wind has spherical symmetry and a constant velocity. It is related to the case when the line is optically thin. The results obtained with the two methods are in good agreement.

In our study we will consider the hot wind in the nebular approach, i.e. we will follow the second of these methods and that is why the value of the mass-loss rate, derived in this case will be used. The arithmetical means of the data of Vogel \& Nussbaumer (1994) and Altamore \& Cassatella (1997) for the years 1986 and 1995 are 1.55 $10^{-7} M_{\odot} \mathrm{yr}^{-1}$ and $8.7510^{-8} M_{\odot} \mathrm{yr}^{-1}$ respectively. The second of them was calculated using the value of Vogel \& Nussbaumer for the year 1993, as their observations were up to this moment.

The line flux determined by recombinations is given by

$F=\frac{h \nu}{4 \pi d^{2}} \int_{V} n_{\text {ion }} n_{\mathrm{e}} \alpha_{\text {eff }}\left(T_{\mathrm{e}}, n_{\mathrm{e}}\right) \mathrm{d} V$

where $n_{\text {ion }}$ is the density of the ion treated; $n_{\mathrm{e}}$, the electron density; $\alpha_{\mathrm{eff}}\left(T_{\mathrm{e}}, n_{\mathrm{e}}\right)$, the effective recombination coefficient of this line and $V$, the emitting volume. If helium is ionized twice in this volume, the electron density will be $n_{\mathrm{e}}=(1+$ $2 a(\mathrm{He})) n$ and the density of the given ion $n_{\text {ion }}=a(X) n$, where $a(X)$ is the abundance by number of the element $X$ relative to hydrogen. Then for the line flux we obtain

$F=\frac{h \nu a(X)(1+2 a(\mathrm{He})) \overline{\alpha_{\mathrm{eff}}}}{4 \pi d^{2}} \int_{V} n^{2} \mathrm{~d} V$.

The abundance by number of He relative to $\mathrm{H}, a(\mathrm{He})$, is adopted to be 0.1 (Vogel 1993; Vogel \& Nussbaumer 1994). The recombination coefficient $\overline{\alpha_{\text {eff }}}$ corresponds to an electron temperature of $T_{\mathrm{e}}=20000 \mathrm{~K}$ (Pottasch 1984). The distance to the system $d$ is assumed to be $650 \mathrm{pc}$, which value is used mostly in the current analyses (Mürset et al. 1991; Vogel \& Nussbaumer 1994; Mürset et al. 1995; Altamore \& Cassatella 1997). The density in the hot wind is a function of the distance to the center and can be expressed via the continuity equation

$n(r)=\frac{\dot{M}}{4 \pi r^{2} \mu m_{\mathrm{H}} v}$,

where $\dot{M}$ is the mass-loss rate; $v$, the wind velocity equal to $1000 \mathrm{~km} \mathrm{~s}^{-1}$ and $\mu m_{\mathrm{H}}$, the mean molecular weight in the hot wind, $\mu=1.4$ (Nussbaumer \& Vogel 1987). Using Eqs. (2) and (3) we can calculate the line flux emitted by a nebula formed by a wind with spherical symmetry and a constant velocity.

We will consider that the temperature of the hot companion of the AG Peg system in both moments has been equal to $90000 \mathrm{~K}$, which is approximately an average of the results of Mürset et al. (1991), Kenyon et al. (1993) and Altamore \& Cassatella (1997). In this case the photon fluxes beyond the limits of the ground series of hydrogen and ionized helium (Nussbaumer \& Vogel 1987) are fully sufficient for ionizing these elements in the hot wind to infinity.

Table 5. The line fluxes of the broad components in units of $10^{-11} \mathrm{erg} \mathrm{cm}^{-2} \mathrm{~s}^{-1}$

\begin{tabular}{lllllr}
\hline \multirow{2}{*}{ Line } & \multicolumn{2}{c}{1986} & & \multicolumn{2}{c}{1995} \\
\cline { 2 - 3 } \cline { 6 - 6 } & $F_{\text {calc }}$ & $F_{\text {obs }}$ & & $F_{\text {calc }}$ & $F_{\text {obs }}$ \\
\hline $\mathrm{H}_{\beta}(\mathrm{B})$ & 2.86 & 2.43 & & 1.12 & 1.61 \\
$\mathrm{H}_{\gamma}(\mathrm{B})$ & 1.36 & 1.95 & & 0.53 & $<0.70$ \\
He II 4686 (B) & 3.12 & 4.35 & & 1.22 & 1.65 \\
\hline
\end{tabular}

It is necessary for the calculation of the line fluxes to determine the region of integration. We treat the wind in the nebular approach (Vogel 1993; Vogel \& Nussbaumer 1994) and that is why the inner radius is thought to be the radius of the star. We will use for its value the arithmetical means of Vogel \& Nussbaumer (1994) and Altamore \& Cassatella (1997), which are $0.11 R_{\odot}$ and $0.09 R_{\odot}$ in the two moments respectively. Let's consider the outer radius. The fact that the radial velocity of the broad components varies with the orbital phase (Tomov \& Tomova 1992) means that their emitting volumes are gravitationaly connected with the star and are not probably extended to a great distance. The calculation of the line fluxes supports this conclusion since the gas at a distances greater than half of the binary separation was found to have no contribution. We will use for this reason an outer radius equal to $300 R_{\odot}$.

The calculated fluxes are listed in Table 5 where they are compared with the observed ones. It is seen that their difference ranges up to about $45 \%$. One of the reasons for this difference is the equivalent width error, which is due to the error of the local continuum. The equivalent width error of the broad components reaches $50 \%$.

\section{Discussion}

It was presented in the work of Tomov \& Tomova (1992) that the orbital variations of the radial velocity of the He II 4686 broad component are opposite in phase with 
those ones of the giant and are determined by the motion of the hot companion. The radial velocity variations of all nebular lines (the lines of ionized metals are an exception) have practically the same phase shift in relation to the radial velocity variations of the two stellar components (Fig. 3). This means the radial velocities of all these lines are determined by the same flow in the circumstellar nebula which, as a whole, is not at rest and there is probably a pronounced large-scale motion in it. The phase shift of the radial velocity curves shows the gas flow is in correlation with the orbital motion. However, the different amplitudes probably indicate some stratification in the direction of the motion causing the various lines to be appear in areas with different velocities.

The described behaviour of the radial velocities during the orbital cycle is related to a binary system, where both components have a stellar wind. Then the two winds must collide head on, as presented in a simplified approximation by Girard \& Willson (1987). A nebular region with a high density and a low velocity of the gas motion is formed as a result of that collision. That is why it was supposed (Tomov 1993b) that the radial velocities of all of the visual narrow lines of AG Peg are probably determined by the motion in the conical part of the nebular region of this system.

The analysis of the emission line spectrum (Sect. 3) showed that the intensity of all lines has decreased. The possible excitation mechanisms in the nebular region include shocks and photoionization. That is why the total flux of the emission lines appearing in this region will consist of two parts: one determined by radiative heating and the other - by shock excitation. The interpretation of the decrease of these fluxes requires modelling in detail of the emitting region. This is a problem of a theoretical nature, including computation of its shape and size as well as the velocity and density distribution. However, it is possible to show by means of elementary calculation that the fluxes would be decreased by a factor close to the mean observed decrease factor of all elements if ionization is realized only by shocks. The mass-loss rate of the giant and the velocity of its wind are considered to be constant having values $210^{-7} M_{\odot} \mathrm{yr}^{-1}$ and $20 \mathrm{~km} \mathrm{~s}^{-1}$ (Vogel \& Nussbaumer 1994; Mürset et al. 1995). The wind parameters of the hot companion are presented in the last section. Then the sum of kinetic energies of the winds in the two moments considered by us is calculated to be $4.9110^{34} \mathrm{erg} \mathrm{s}^{-1}$ and $2.7710^{34} \mathrm{erg} \mathrm{s}^{-1}$ respectively. These are upper limits, since the wind velocity components normal to the nebular region surface in its predominant part are less than the velocisties themselves. The ratio of these amounts is 1.8 and the average of the observed values of the decrease factor of the different elements is 2.5 .

\section{Conclusions}

We present results of observations of the blue spectrum of the symbiotic system AG Peg at the final stage of its outburst when the luminosity and the mass-loss rate of its hot component decrease. The radial velocity data and the line fluxes have been obtained.

The radial velocities of the emission lines are compared with the radial velocity curves of the same elements during the orbital cycle, drawn on the basis of earlier observations, realized nine years ago (Tomov \& Tomova 1992). It turns out that some of them differ considerably from the corresponding values of the curves, which in our opinion, is determined by dynamical change of the nebular environment. Balmer progression has not been established.

The profile of the lines $\mathrm{H} \beta, \mathrm{H} \gamma$ and $\mathrm{He}$ II 4686 consists of two components: a central narrow component and a broad component indicating stellar wind. The comparison with earlier spectra (Tomov \& Tomova 1992) shows the width of the broad component has not changed, which means that the wind velocity has had a constant value. The line fluxes of the broad components have been calculated by means of the mass-loss rate, obtained on the basis of contemporaneous UV observations. They are in agreement with the observed fluxes which confirms this mass-loss rate and its decreasing by a factor of 1.77 during this period of nine years.

The line fluxes of the more intensive among the narrow lines have also been obtained. A decrease of these fluxes by a mean factor of about 2 for all elements has been derived during this period.

Acknowledgements. The authors are grateful to the referee whose critical remarks contributed to improve the paper. N.T. and M.T. would like to thank their colleagues Dr. R. Zamanov and Dr. N. Markova for helpful discussions. This work was supported in part by Bulgarian National Scientific Foundation grant under contract F-466/94 with Ministry of Education, Science and Technology.

\section{References}

Altamore A., Cassatella A., 1997, A\&A 317, 712

Boyarchuk A.A., 1966, AZh 43, 976

Boyarchuk A.A., 1967, AZh 44, 12

Fernie J.D., 1985, PASP 97, 653

Girard T., Willson L.A., 1987, A\&A 183, 247

Hutchings J.B., Redman R.O., 1972, PASP 84, 240

Hutchings J.B., Cowley A.P., Redman R.O., 1975, ApJ 201, 404

Ilmas M., 1987, in: Observations and Interpretation of Stellar Spectra. Dushanbe, p. 81

Kenyon S.J., Mikolajewska J., Mikolajewski M., Polidan R.S., Slovak M.H., 1993, AJ 106, 1573 
Lundmark K., 1921, Astron. Nachrichten 213, Nr. 5094, 93 Mürset U., Nussbaumer H., Schmid H.M., Vogel M., 1991, A\&A 248, 458

Mürset U., Jordan S., Walder R., 1995, A\&A 297, L87

Nussbaumer H., Vogel M., 1987 A\&A 182, 51

Penston M.V., Allen D.A., 1985, MNRAS 212, 939

Pottasch S.R., 1984, Planetary nebulae. Reidel, Dordrecht, p. 45

Seaton M.J., 1979, MNRAS 187, 73p
Schmutz W., Hamann W.-R., Wessolowski U., 1989, A\&A 210, 236

Tomov N.A., 1993a, Izv. Krym. Astrofiz. Obs. 88, 11

Tomov N.A., 1993b, Izv. Krym. Astrofiz. Obs. 88, 22

Tomov N.A., Tomova M.T., 1992, Izv. Krym. Astrofiz. Obs. 86,19

Vogel M., 1993, A\&A 274, L21

Vogel M., Nussbaumer H., 1994, A\&A 284, 145

Zamanov R.K., Tomov N.A., 1995, Observatory 115, 185 